\title{
THE PRODUCTION OF ECOFRIENDLY BIOFILM WITH NATURAL OIL FOR FOOD PACKAGING
}

\author{
Emine Arman Kandirmaz ${ }^{1}$ (D), Omer Bunyamin Zelzele ${ }^{2}$ \\ ${ }^{1}$ Marmara University, School of Applied Sciences, Printing Technologies, Istanbul, Turkey \\ ${ }^{2}$ Marmara University, Vocational School of Technical Sciences, Printing and Publishing \\ Technologies, Istanbul, Turkey
}

\begin{abstract}
The use of edible biofilms in food packaging reduces the use of petrochemical polymers that are harmful to human health, such as PE, PP, PET. The second most common biopolymer in nature, chitosan is a nontoxic, nonantigenic, biocompatible and biodegradable polymer. Considering these features, it is frequently used in food packaging applications. Increasing needs for food amount and quality canalized food industry to fund in new packaging techniques that improve storage life and grade of foods. Active packaging systems, one of these methods, can be designed as a sensor, antimicrobial or antimigrant in order to extend the shelf life of the food product and to inform the shelf life in possible degradation. Essential oils, which are antimicrobial environmentally friendly packaging material additives, are used due to their effective biological activities. Essential oils that have known antimicrobial properties include lavender, rosemary, mint, eucalyptus and geranium. These oils are also edible. In this study, it is aimed to produce antimicrobial, ecofriendly, edible, printable biofilm for active packaging, using chitosan and peppermint essential oil. For this purpose, chitosan biofilms containing different rates $(0,1,2.5,5,10 \%)$ of peppermint essential oil were produced by solvent casting method. Surface morphology were examined by SEM. The transparency of biofilms was determined by UV spectroscopy. Antimicrobial properties of the obtained films were determined against S. aureus and E. coli. Biofilms were printed with screen printing. The color, gloss, contact angle, surface tension values of all printed and unprinted samples were examined. As a result, chitosan biofilms which are loaded with peppermint essential oil were successfully produced. Biofilms are colorless, highly transparent and have good printability. It is concluded that the amount of peppermint essential oil increased inhibitory feature against S. aureus and E. coli. When the obtained results are examined, it is determined that the printable, ecofriendly, edible biofilms can be used in active food packaging applications.
\end{abstract}

Keywords: Biofilm, Edible, Printability, Antimicrobial, Peppermint oil

\section{INTRODUCTION}

Food packages basically provide barrier and protective functions to protect food against the environmental conditions, fungal, and bacteria. Packaging industry is developing and advancing in order to meet these conditions. These developments are in the direction of being cheap, healthy, bio-sourced, lightweight, printable and protective, keeping food fresh for longer. Packages should be printable enough to present the final product well enough. For this purpose, packages play not only a passive promotion role, but also play active roles such as preserving food and extending shelf life in many different areas with newly developed types. Active packaging; They are auxiliary packaging materials added to the packaging to improve the use of food (Zhong et al., 2020) Different agents such as enzymes, natural materials or bacteriocins can be added into packaging which are used different materials papers, films etc. (Han, 2003). Some useful flavoring agents and important substances for food preservation such as antioxidant and antimicrobials can be incorporated into packaging systems to be released into food products during their storage instead being used in food formulations as ingredients or additives. This technique allows the release of active substances in the correct amount, gradually during storage time and in the place where more useful (Dutta et al., 2009).

Antimicrobial active packaging can be achieved in by incorporating antimicrobial agents directly into packaging polymers and by using polymers that are antimicrobial by themselves. The most commonly antimicrobials are used in packaging, natural essential oils, enzymes, silver ions and organic acids (Corrales et al., 2014). Peppermint plant is a member of the Labiatae family and has been frequently used in the pharmaceutical industry since ancient times due to its carminative and antiseptic properties (Herrmann and Kucera, 1967). Peppermint oil is effective against many known bacteria and fungi. These include fungi 
such as Candida albicans, C. tropicalis and gram-negative and positive bacteria such as Aspergilus niger, Xanthamonas campestris, Escherichia coli, Salmonella typhimurium (Chao et al., 2000). According to studies on Gram-negative bacteria, the oil obtained from the leaf parts of mint has higher efficiency than the oil obtained from the stem part. In addition, the sensitivity of mint to gram negative bacteria is higher than gram positive (McKay and Blumberg, 2006). Having a high antioxidant effect, peppermint oil can also be used against viruses such as influenza and herpes (Schuhmacher et al., 2003).

In recent years, synthetic polymers have begun to be used more than other materials in food packaging due to reasons such as ease of production, lightness, cheapness and accessibility (Tajeddin and Arabkhedri, 2020). According to the needs of the material to be packaged; The production of a wide variety of packaging materials is another factor in increasing usage. With the decrease in oil, which is the main raw material of polymers, the increase in price and the problems in getting rid of its waste, the interest in environmentally friendly natural polymers increases. In this respect, interest in natural and biodegradable packaging materials that can be used in food packaging is increasing and its production becomes essential. Natural polymers are described as macromolecules which are completely or partially obtained from renewable plants or animals. Bio-based polymers are generally classified three categories: natural polymers extracted from animal or plant sources (starch, cellulose, casein), chemical synthisized polymers which are produced by renewable bio-based monomers (polylactate), genetically modified bacteria's produced polymers (polyhydroxy alkanoates). Chitosan is a natural polymer that is usually made from shellfish. Chitosan has been proven in many studies to have antibacterial properties (Yaday et al., 2019). It can be used in active packaging applications due to its ability to be made into a film structure, and its natural antibacterial properties. Chitosan biofilms can be a good packaging material due to their low permeability and high barrier against oil, but their low resistance to water limits their usage areas.

In this study, it is aimed to produce edible, environmentally friendly biofilm that can be used in food packaging using chitosan and peppermint oil. Essential oil and biopolymer were chosen for their antimicrobial properties.

\section{METHODS}

The mint samples obtained from Yalova region were weighed $200 \mathrm{~g}$ from the sample after drying at $30{ }^{\circ} \mathrm{C}$. These samples were then subjected to hydro distillation for 6 hours with the NeoClevenger apparatus. Essential oils were dried with anhydroussodiumsulfate and stored in dark bottles and stored at $4{ }^{\circ} \mathrm{C}$.

Chitosan blank films (FO) were produced with a solvent casting method. A solution of chitosan $(0.5 \% \mathrm{w} / \mathrm{v})$ was prepared by dissolving $1.0 \mathrm{~g}$ of chitosan in $200 \mathrm{~mL}$ of deionized water/acetic acid (2\%) at $25^{\circ} \mathrm{C}$ under stirring for 3 hours $750 \mathrm{rpm}$. After complete dissolution, PEG $(0.2 \mathrm{~g})$ was added as a plasticizer to increase film flexibility, decrease fragility and to simplified film separation from petri. The obtained bio based polymeric film solution was spilled onto petri that $9 \mathrm{~cm}$ diameter and dried in vacuum at $30{ }^{\circ} \mathrm{C}$ for a day. The films were removed from the petri. Similar to the procedure described above, 1, 2.5, 5, 10\% peppermint oil was added into the same formulation and mixed in an ultrasonic mixer for 5 minutes at room temperature and F1,2,3,4 films were produced. The contents of all films are given in Table 1.

Table 1: The film formulations

\begin{tabular}{|c|c|c|c|}
\hline Formulation No & Chitosan & PEG & Peppermint Oil \\
\hline F0 & 83 & 17 & 0 \\
\hline F1 & 83 & 17 & 1 \\
\hline F2 & 83 & 17 & 2.5 \\
\hline F3 & 83 & 17 & 5 \\
\hline F4 & 83 & 17 & 10 \\
\hline
\end{tabular}

Surface morphology were examined by SEM with Phillips XL 30 ESEM-FEG microscope. Bio based films were cryo-fractured with liquid nitrogen. Fractured films surfaces coated with gold before SEM imaging. The transparency of biofilms was determined by Shimadzu UV-vis spectrophotometer 2450 (Kyoto Jap). Antimicrobial properties of the obtained films were determined against S. aureus and E. coli. Antimicrobial 
activity of films was defined using the inhibition zone (disc diffusion) method. Each bacterial culture was activated, by inoculation in Tryptic Soy Broth (TSB) at $37{ }^{\circ} \mathrm{C}$ for 24 hours. Inoculum $(0.1 \mathrm{~mL})$ was spread over the Mueller-Hinton ( $\mathrm{MH}$ ) agar petri plates, then 4-mm-wide films were placed into the petri plates. The disc film involving the petri plates and control samples were incubated at $37^{\circ} \mathrm{C}$ for 24 hours. Following the incubation process, the petri plates were examined for bacterial growth and the inhibition zones around the disc films were assessed in qualitative and quantitative terms. Quantitative assessment was performed based on the inhibition zone diameter. The zones around the disc films were assessed as the indicator of the inhibition of bacterial growth.

All films were printed with Process Magenta (TOYO ink) commercial screen printing ink using an ARUS semiautomatic printability test device (Printing parameters; weaving density of $77 \mathrm{tpc}, 75$ degree scraping angle and 75 degree shore hardness). CIE L*a*b* rates of all films were determined with X-Rite eXact spectrophotometer in accordance with ISO 12647-2:2013, (spectral range $400-700 \mathrm{~nm}$, with D50 light, 2 observer angle, with polarize filter, 0/45-degree). The color differences formula was dedicated below. All calculations were made with 5 repetitions.

$\Delta E_{00}=\sqrt{\left(\frac{\Delta L^{\prime}}{k_{L} S_{L}}\right)^{2}+\left(\frac{\Delta C^{\prime}}{k_{C} S_{C}}\right)^{2}+\left(\frac{\Delta H^{\prime}}{k_{H} S_{H}}\right)^{2}+R_{T} \frac{\Delta C^{\prime}}{k_{C} S_{C}} \frac{\Delta H^{\prime}}{k_{H} S_{H}}}$

Gloss values were measured by BYK-Gardner GmbH glossmeter according to ISO 2813:2014 with 60 geometry. Contact angle and total surface energies of the biofilms were determined with Pocket Goniometer PGX+ in line with ASTM D5946.

\section{RESULTS AND DISCUSSION}

Figure 1 demonstrate that the SEM micrographs of the fracture surface morphology of biofilms. Figure 1 shows that polymeric biofilm was a uniform and non-cracked surface.

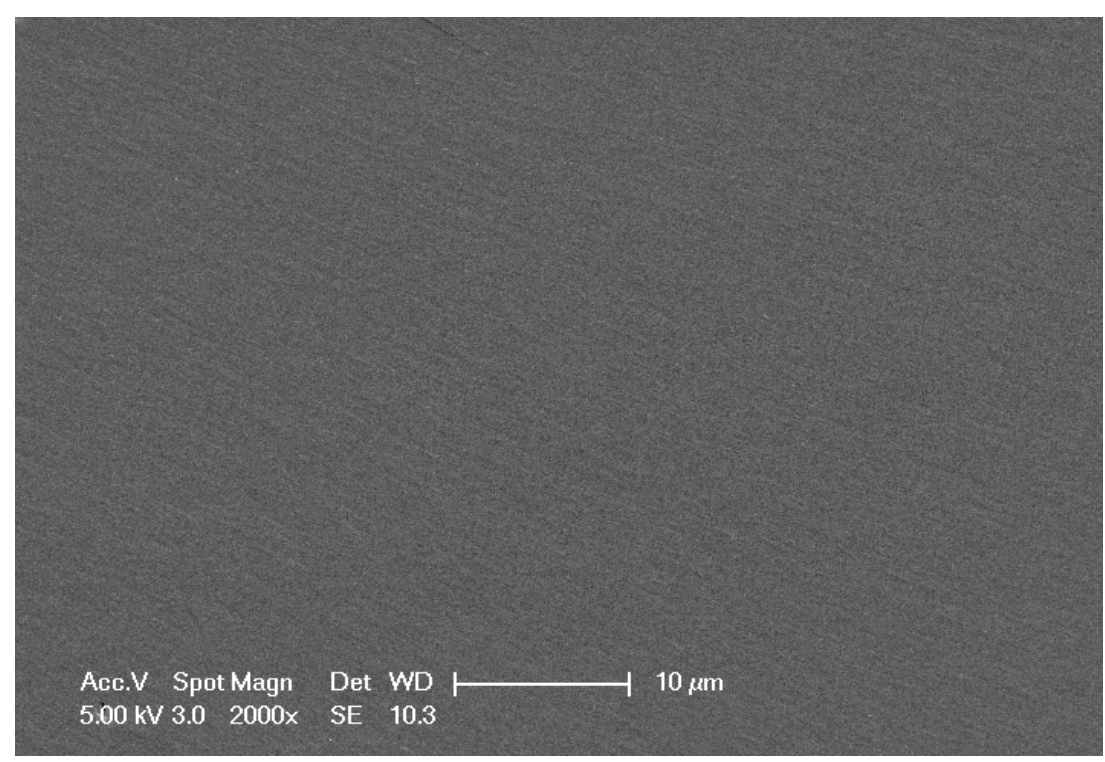

Figure 1: SEM image of F4

Chitosan containing biofilm was transparent. The mint oil containing films were also found to transparent. The optical properties of biofilms were investigated with measuring of transmittance among 300-800 nm. The transmittance spectrums of bio films transmittance value of the films at $750 \mathrm{~nm}$ are 99,5 (\%T). This results was observed that the addition of pepper mint oil causes a negligible decrease transmittance of the chitosan biofilms. 

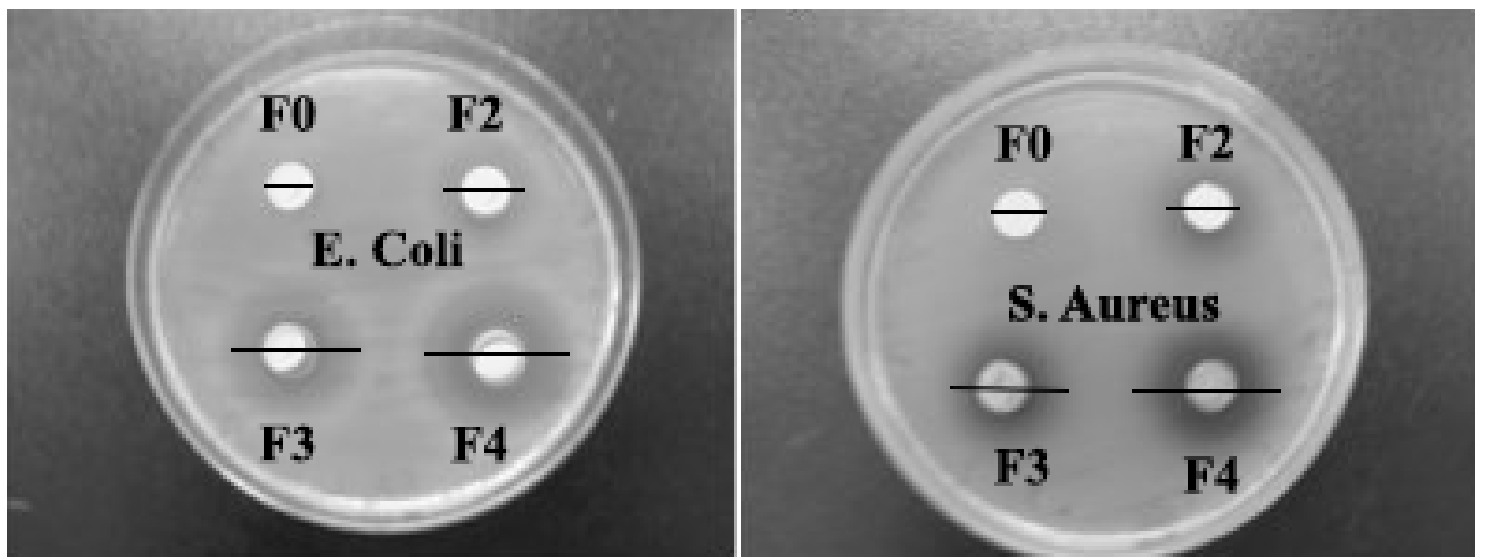

Figure 2: Antimicrobial activity of films using the inhibition zone (disc diffusion) method

Table 2: The antibacterial activity of biofilms

\begin{tabular}{|c|c|c|}
\hline $\begin{array}{c}\text { Formulation } \\
\text { No }\end{array}$ & $\begin{array}{c}\text { S. aureus diameter of } \\
\text { inhibition zone }(\mathrm{mm})\end{array}$ & $\begin{array}{c}\text { E. coli diameter of } \\
\text { inhibition zone }(\mathrm{mm})\end{array}$ \\
\hline F0 & 5 & 5 \\
\hline F2 & 14 & 12 \\
\hline F3 & 20 & 18 \\
\hline F4 & 24 & 22 \\
\hline
\end{tabular}

In bio films include solely chitosan no respectable inhibition zone was considered versus Escherichia coli and Staphylococcus aureus. This result may be concerned to the fact that chitosan does not diffuse owing to neighboring agar environment in the agar diffusion test method, thus only bacteia's in directly touch with the real medium of chitosan are inhibited. As regards chitosan added pepper mint oil, this bio film demonstrated an inhibitive impact for four concentrations, onto 2 bacteria type experienced as well inhibitory effect was concentration-dependent. From the two microorganisms compared, it is understood from the inhibition diameters that the inhibition effect of peppermint oil is more pronounced on S. aureus. The white substrate was used in the measurements and the color values of the films are given in Table 3.

Table 3: Color and gloss properties of biofilms

\begin{tabular}{|c|c|c|c|c|c|}
\hline Formulation No & $\mathrm{L}^{*}$ & $\mathrm{a}^{*}$ & $\mathrm{~b}^{*}$ & $\Delta \mathrm{E}_{00}$ & Gloss \\
\hline White Substrate & 96.4 & 2.8 & -7.3 & Ref. & 5.2 \\
\hline F0 & 96.2 & 2.7 & -5.9 & 1.09 & 28.9 \\
\hline F1 & 96.3 & 2.8 & -5.2 & 1.68 & 28.6 \\
\hline F2 & 96.5 & 2.9 & -4.9 & 1.99 & 28.6 \\
\hline F3 & 96.4 & 2.87 & -4.3 & 2.46 & 28.5 \\
\hline F4 & 96.3 & 2.81 & -4.1 & 2.62 & 28.4 \\
\hline
\end{tabular}


Color differences were calculated by delta $\mathrm{E}$ of films (F0, F1, F2, F3, F4) how it differs from white substrate. When the table is examined, it is concluded but only biofilms shifted the color slightly to yellow but also Bio films did not variety the color much. $\Delta E$ difference did not notice with eye because of color difference was below 3. It is also within the acceptable reference range according to ISO 12647-2. When the gloss values were examined, it was determined that all films were at least five times glossier than the base paper. This is due to the fact that the roughness on the paper surface is covered by the coating and the diffuse reflection is prevented. Thus, the papers became glossier.

Surface energy plays an important role in the interaction of printing process. The content of the substrate is among the parameters that affect surface, contact angle and surface energy. The contact angles and surface energies of the obtained coatings are given in Table 4.

Table 4: Total surface energy and Contact Angle values of biofilms according to ASTM D5946 method

\begin{tabular}{|c|c|c|c|}
\hline Formulation No & $\begin{array}{c}\text { Surface } \\
\text { Energy } \\
\text { (mJ/m2) }\end{array}$ & $\begin{array}{c}\text { Contact } \\
\text { Angle }\end{array}$ & Image \\
\hline F0 & 38.9 & 72.3 & \\
\hline F1 & 37.3 & 76.8 & \\
\hline F2 & 36.4 & 79.1 & \\
\hline F3 & 35.2 & 82.5 & \\
\hline F4 & & 83.9 & \\
\hline
\end{tabular}

The water contact angle of FO was found as $72.3^{\circ}$. When pepper mint oil was added to base formulation, the contact angle increased to $76.8^{\circ}$. This is due to the hydrophobic nature of the oil. It is seen that the contact angle increases when the amount of oil in the biofilm formulation is increased. In all biofilms, contact angle values were higher than F0. This improves the offset and screen printing printability of the biofilms with less ink. Because the oleophilic groups in the offset and screen printing ink structure will interact more with the peppermint oil on the biofilm surface and a good ink surface relation will be provided and the printing process will be easier.

Screen printing test prints were made with ARUS printing test machine on the all biofilms. CIEL*a*b* color, gloss, color differences are given in Table 5. When Table 5 is examined, it is determined that the color has shifted to yellow a little, as in unprinted films, it changes the color a little. However, the color difference is lower than the films and is too small to be detected by the human eye. As the amount of oil increases, the color difference increases slightly. This shows that the ink tolerates the color difference of the magenta tone. When the gloss results were examined, the results were obtained in the same direction with the unprinted films. However, it has been determined that due to the solid particles in the ink roughening the surface, it has been determined that there is a proportional decrease in gloss in all films by $1 / 3$. 
Table 5: Color and gloss properties of printed biofilms

\begin{tabular}{|c|c|c|c|c|c|c|}
\hline Formulation No & $\mathrm{L}^{*}$ & $\mathrm{a}^{*}$ & $\mathrm{~b}^{*}$ & $\Delta \mathrm{E}_{00}$ & Image & Gloss \\
\hline F0 & 47.7 & 74.2 & -3.5 & Ref. & & 16.9 \\
\hline F1 & 47.8 & 74.6 & -1.4 & 1.02 & & 16.2 \\
\hline F2 & 47.9 & 74.5 & -1.2 & 0.92 & & 16.3 \\
\hline F3 & 47.8 & 74.8 & -0.9 & 1.45 & & 16.3 \\
\hline F4 & 47.6 & 74.7 & -0.8 & 1.44 & & \\
\hline
\end{tabular}

\section{CONCLUSIONS}

As a result, peppermint oil containing chitosan-based biofilms have been successfully prepared. The obtained films have uniform surface and transparent. Biofilms containing peppermint oil have inhibitory properties against both gram positive and negative bacteria. The highest activity was observed against s.aureus. When the colors of the films were examined, it was determined that the color difference was below 3, that is, there is no color difference that can be seen by the eye. With increased contact angle of oil-containing biofilms, the surface has become more sensitive to oil-based ink. The printability of the biofilm is increased by adding peppermint oil. Screen prints were made on biofilms without any problem.

\section{REFERENCES}

[1] Chao, S. C., Young, D. G., Oberg, C. J.: "Screening for inhibitory activity of essential oils on selected bacteria, fungi and viruses", Journal of essential oil research 12 (5), 639-649, 2000. doi: 10.1080/10412905.2000.9712177.

[2] Corrales, M., Fernandez, A., \& Han, J. H.: “Antimicrobial packaging systems. In Innovations in food packaging" (, Academic Press, 2014.), pages 133-170.

[3] Dutta, P. K., Tripathi, S., Mehrotra, G. K., Dutta, J.: "Perspectives for chitosan based antimicrobial films in food applications", Food chemistry 114 (4), 1173-1182, 2009. doi: 10.1016/j.foodchem.2008.11.047.

[4] Han, J. H.: "Innovations in food packaging" (Academic Press, Cambridge MA, 2014.), pages 133-170.

[5] Herrmann Jr, E. C., Kucera, L. S.: "Antiviral Substances in Plants of the Mint Family (Labiatae). III. Peppermint (Mentha piperita) and other Mint Plants", Proceedings of the Society for Experimental Biology and Medicine 124 (3), 874-878, 1967. doi: 10.3181/00379727-124-31874.

[6] McKay, D. L., Blumberg, J. B.: "A review of the bioactivity and potential health benefits of peppermint tea (Mentha piperita L.). Phytotherapy Research", An International Journal Devoted to Pharmacological and Toxicological Evaluation of Natural Product Derivatives 20 (8), 619-633, 2006. doi: 10.1002/ptr.1936.

[7] Schuhmacher, A., Reichling, J., \& Schnitzler, P.: "Virucidal effect of peppermint oil on the enveloped viruses herpes simplex virus type 1 and type 2 in vitro", Phytomedicine 10 (6-7), 504-510, 2003. doi: 10.1078/094471103322331467.

[8] Tajeddin, B., \& Arabkhedri, M.: "Polymers and food packaging." In Polymer Science and Innovative Applications (pages 525-543). Elsevier, 2020. doi: 10.1016/B978-0-12-816808-0.00016-0

[9] Yadav, M., Goswami, P., Paritosh, K., Kumar, M., Pareek, N., Vivekanand, V.: "Seafood waste: a source for preparation of commercially employable chitin/chitosan materials", Bioresources and Bioprocessing 6 (1), 8, 2019. doi: 10.1186/s40643-019-0243-y. 
[10] Zhong, Y., Godwin, P., Jin, Y., \& Xiao, H.:"Biodegradable polymers and green-based antimicrobial packaging materials: A mini-review.", Advanced Industrial and Engineering Polymer Research 3 (1), 27-35, 2020. doi: 10.1016/j.aiepr.2019.11.002

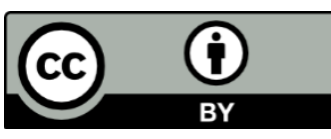

(C) 2020 Authors. Published by the University of Novi Sad, Faculty of Technical Sciences, Department of Graphic Engineering and Design. This article is an open access article distributed under the terms and conditions of the Creative Commons Attribution license 3.0 Serbia (http://creativecommons.org/licenses/by/3.0/rs/). 